\title{
Dysbiosis of Gut Microbiota and Acne Vulgaris: The Role of Short- Chain Fatty Acids Gut in Pathogenesis
}

\section{Abdelhakim $\mathrm{M}^{1}$, Maeno $\mathrm{T}^{2}$, Ozaki $\mathrm{D}^{3}$ and Hitosugi $\mathrm{N}^{1 *}$ \\ ${ }^{1}$ Muse Dermatology \& Pain Clinic, Musashiurawa Medical Center, Japan \\ ${ }^{2}$ Musashi Pharmacy, Musashiurawa Medical Center, Japan \\ ${ }^{3}$ Musashiurawa Orthopedics \& Internal Medicine Clinic, Musashiurawa Medical Center, Japan}

*Corresponding author: Naoko Hitosugi, M.D., Ph.D., Muse Dermatology \& Pain Clinic, 7 Chome-2-1 Bessho, Minami Ward, Saitama 336-002, Japan, Tel: +81-4-8866-4112; Fax: +81-48866-4371; Email: 634muse@gmail.com

\section{Research Note \\ Volume 5 Issue 3}

Received Date: August 26, 2020

Published Date: September 18, 2020

DOI: $10.23880 /$ cdoaj-16000218

\section{Abstract}

Introduction: Acne vulgaris (AV) is a common skin disease caused by chronic inflammation characterized by overproduction of sebum and secretion of inflammatory cytokines in the skin. Recent studies suggest a link between dysbiosis of the gut microbiota and the development of inflammatory skin diseases caused by reduced production of immunomodulators. Vitamins have been also reported to play an essential role in regulating microbiome dynamics. Therefore, we hypothesized that gut microbiota deposition alters short chain fatty acid (SCFAs) production which regulate the permeability of gut barrier.

Method: In this study, patients between 20-25 years old with or without acne were recruited. Fecal samples were collected to analyze the fecal microbiota composition and the concentration of short chain fatty acids (SCFAs) were measured by gas chromatography. In addition, blood levels of vitamins including; riboflavin (vitamin B2), and pyridoxine (Vitamin B6) were assessed.

Result: At the phylum level, the abundance of Firmicutes was lower, but Bacteroidetes was higher in patients with acne. At genus level, Fecalibacterium was distinctly higher in abundance in the AV microbiota. A significant difference $(P<0.05)$ in Vitamin B2 (Riboflavin) was found between the high-level Fecalibacterium and low-level Fecalibacterium groups. Analysis of fecal SCFAs showed a significant difference between acetate concentration and AV microbiota.

Conclusion: Our findings suggest that Vitamin B2 and SCFAs, particularly acetates, are essential in maintaining the balance of gut microbiota. Failing to maintain the balance of certain gut microbiota such as Fecalibacterium may result in stimulating gut epithelial inflammation and release of harmful substances to reach the skin resulting in further skin irritation and the development of acne vulgaris. Further investigations on the relationship between gut microbiota dysbiosis and acne vulgaris may provide effective diagnosis and therapy for this disorder.

Keywords: Acne vulgaris; Inflammation; Fecalibacterium; Gut microbiota 


\section{Clinical Dermatology Open Access Journal}

\section{Research Note}

Acne vulgaris (AV) is a common skin disease caused by chronic inflammation of the pilosebaceous units and often affects adolescents, particularly in developed countries. Overproduction of sebum, secretion of inflammatory cytokines in the skin, and follicular hyperkeratinization of pilosebaceous ducts characterize the pathogenesis [1].

Up to 100 trillion microbes, representing more than 1000 species of intestinal bacteria, coexist in the human intestine, and their association with several diseases such as chronic diseases including atopic dermatitis (AD), Crohn's disease and obesity has been reported in the human microbiome in a non-causal manner [2].

Recent studies suggest a link between dysbiosis of the gut microbiota and the development of inflammatory skin diseases with dysfunction of the intestinal barrier. For instance, acne vulgaris has been reported to be significantly linked with irritable bowel syndrome patients suggesting an underlying organic pathology [3]. Particularly, a recent study has investigated the phenomenon of disordered gut microbiota in patients with acne and suggested it as a contributor underlining the disease [4].

The alteration in gut microbial environment has been reported to cause reduction production of immunomodulators, specifically short-chain fatty acids (SCFAs), which are known to have anti-inflammatory effects that contribute to the maintenance of epithelial barrier function [5]. Furthermore, vitamins have been reported to play an essential role in regulating microbiome dynamics and highlighted studies the importance of vitamin acquisition for microbes in the gut environment. Among various contributing vitamins; cobalamin (Vitamin $\mathrm{B}_{12}$ ) is the bestknown example [6].

To date, the evidence supporting the relationship between dysbiosis and the pathogenesis of skin disorders is insufficient. Thus, to further investigate the hypothesis that the imbalance of gut microbiota contributes to the pathogenesis of acne vulgaris, here, in this study we analyzed the fecal microbiota composition of 20 female Japanese patients with AV (AV microbiota) aged between 20 and 25 years, and compared them with that of age-matched healthy controls (non-AV microbiota) using 16S rRNA gene sequencing. Next, SCFA concentration in fecal samples was measured and comparatively analyzed by using gas chromatography-mass spectrometry; the major SCFAs such as; acetate, propionate, butyrate, and valerate were selected for analysis. In addition, blood levels of vitamins including; riboflavin (vitamin $\mathrm{B}_{2}$ ), and pyridoxine (Vitamin $\mathrm{B}_{6}$ ) were assessed.
None of the subjects received antibiotic treatment within 6 months before fecal sample collection. The severity of acne vulgaris was assessed by the number of inflammatory lesions on half of the face, according to the Japanese Acne Study Group criteria [7].

The study protocol complied with the World Medical Association's Declaration of Helsinki 1964, and its later amendments. Written informed consent was obtained from all participants.

At the phylum level, the abundance of Firmicutes was lower, but Bacteroidetes was higher in patients with acne. However, no significant difference was observed in the microbial $(\alpha)$ diversity of all AV microbiota compared with that of all non-AV microbiota. Likewise, the dissimilarity in composition between the $\mathrm{AV}$ and non-AV microbiota ( $\beta$ diversity) was not significant (data not shown). At genus level, Fecalibacterium was distinctly higher in abundance in the AV microbiota, and thus we further investigated the relationship between the disordered Fecalibacterium with vitamin levels of the patients. A significant difference $(\mathrm{P}<$ 0.05 ) in Vitamin $B_{2}$ (Riboflavin) was found between the highlevel Fecalibacterium and low-level Fecalibacterium groups (Figure 1a).

Furthermore, analysis of fecal SCFAs showed a significant difference between acetate concentration and AV microbiota; there were no significant differences in butyrate, propionate, and total lactate levels, or in the ratio of iso- and n-butyrate/valerate levels (Figure 1b). Because the total level of Fecalibacterium in these samples was much higher than the levels of other SCFA-producing bacteria, the SCFA profile results could be directly linked to changes in Fecalibacterium composition.

In this study, patients with acne had a lower abundance of Firmicutes and higher Bacteroidetes when compared with healthy controls. This increased ratio of Bacteroidetes to Firmicutes has been reported to be associated with some inflammatory disorders [4]. In parallel, in a previous in vitro study, Fecalibacterium growth was reported to be highly stimulated in the presence of acetate [8], which goes in line with the present study findings.

Our study provides a supporting evidence to the hypothesis that the gut microbiome influence skin microbiome through a diversity of mechanisms; first, SCFAs resulting from fiber fermentation in the gut - propionate, acetate, and butyrate - may play a pivotal role in determining the predominance of certain gut microbiome profiles which subsequently influence cutaneous immune defense mechanisms [9]. 
A

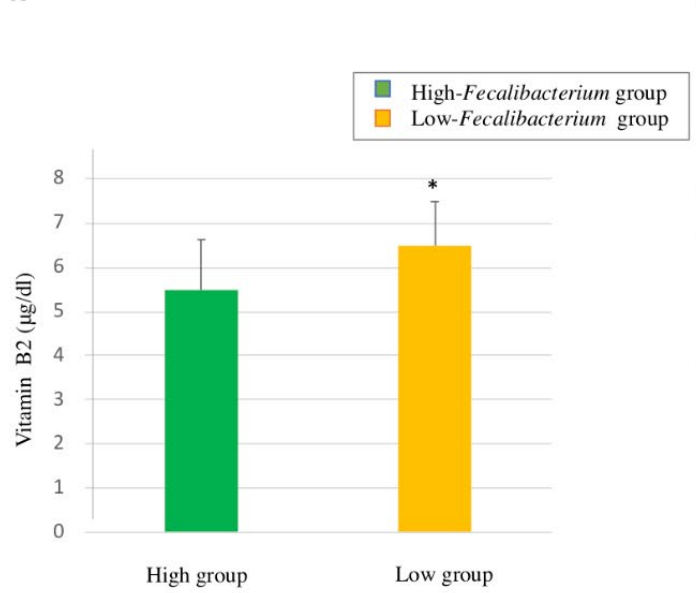

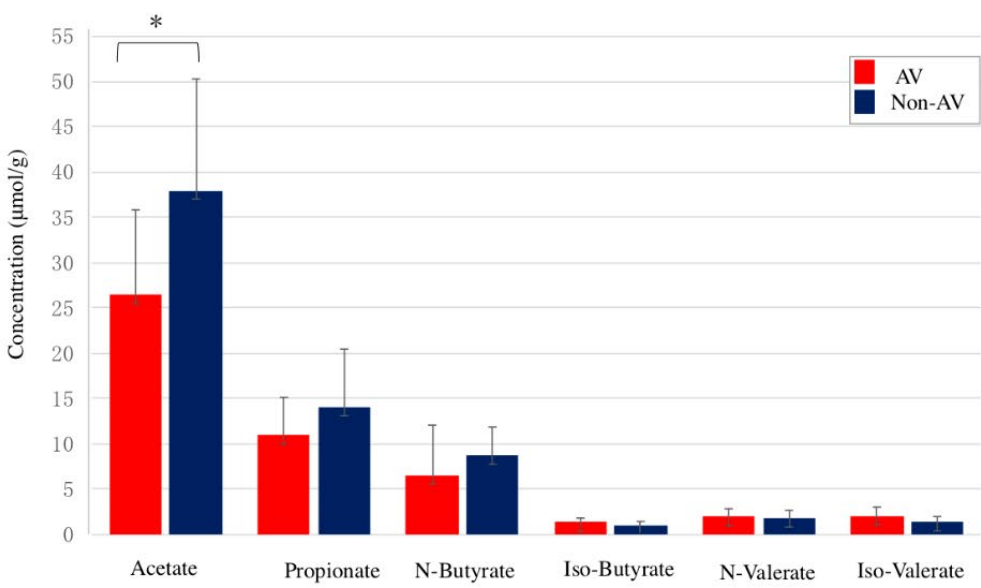

Figure 1A: Graph representing the analysis of high- and low-level Fecalibacterium groups in association with vitamin $B_{2}$ levels. A significant difference ${ }^{*}(P<0.05)$ in vitamin $B_{2}$ (Riboflavin) level was found between the high-level and low-level Fecalibacterium groups. The high-level group comprised those with values above the mean $(>0.75954)$, and the low-level group comprised those with values below the mean $(<0.75954)$.

Figure 1B: Graph representing analyses of SCFAs in fecal samples. Gas chromatography-mass spectrometry quantification of selected SCFAs in AV and non-AV fecal samples. ${ }^{*}(P<0.05)$, the Mann-Whitney U test was used to analyze significance; $\mathrm{P}<0.05$ was considered significant.

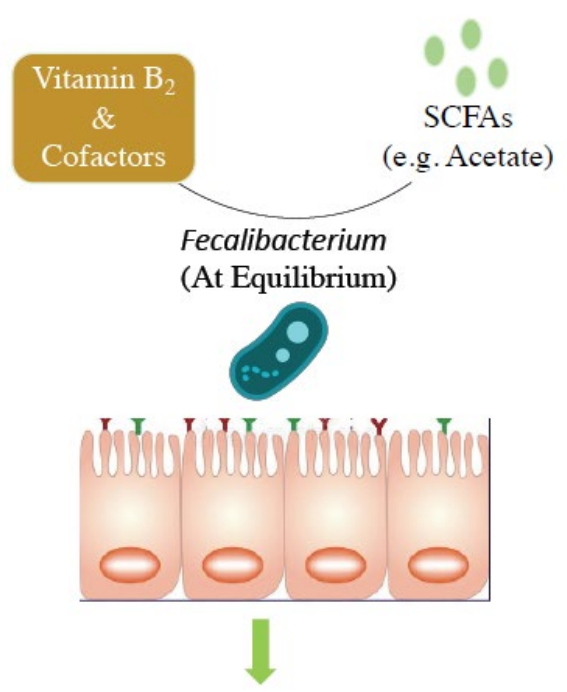

Intact gut epithelial barrier

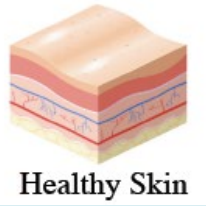

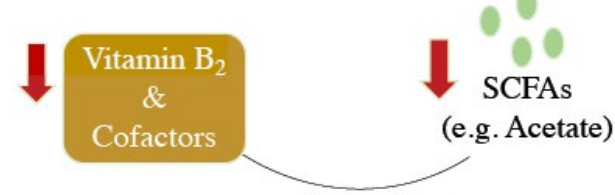

Fecalibacterium

(Disturbed ratio in Gut)

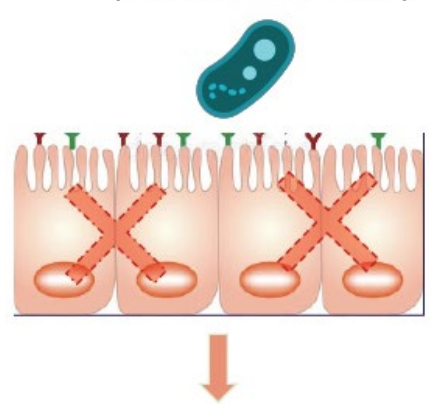

Released toxins causing skin inflammation

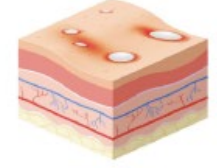

Acne Vulgaris

Figure 2: An illustrative graphic demonstrating the proposed pathogenesis in acne vulgaris caused by the lack of Vitamin $B_{2}$ and SCFAs, particularly acetates that are essential in maintaining the balance of gut microbiota in the healthy human gut such as Fecalibacterium. Thus, resulting in stimulating gut epithelial inflammation and releasing harmful substances to that further irritate the skin. 


\section{Clinical Dermatology Open Access Journal}

Second, studies have suggested that riboflavin (vitamin $\mathrm{B}_{2}$ ) may act as an important factor benefitting Faecalibacterium prausnitzii and proposed that a riboflavinrich diet may promote the regulation of the adequate abundance of Fecalibacteria in the human gut [10].

In this study, we were able to detect specific characteristics in gut microbiota diversity in AV patients specifically in Fecalibacterium associated with reduced levels of Vitamin $\mathrm{B}_{2}$ and increased fecal acetate levels. However, this study has several limitations including: the small number of the involved patients and the limited locality of the subjects thus, the risk of bias may exist among the considered cases; and finally, the study only described the phenomenon of disordered gut microbiota in patients with acne vulgaris. Therefore, further studies are needed to investigate the pathogenesis of microbiota underlining acne vulgaris.

In conclusion, our findings suggest that Vitamin $B_{2}$ and SCFAs, particularly acetates, are essential in maintaining the balance of gut microbiota in the healthy human gut. Failing to maintain the balance of certain gut microbiota such as Fecalibacterium, due to dysregulation of essential cofactors may result in stimulating gut epithelial inflammation and allowing harmful substances to reach the skin via a phenomenon that has been described as "leaky gut syndrome" [11], resulting in further skin irritation and the development of acne vulgaris as illustrated in (Figure 2). Further investigations on the relationship between gut microbiota dysbiosis and acne vulgaris are warranted, and may provide effective diagnosis and therapy for this disorder.

\section{Acknowledgements}

We thank the patients for their enthusiastic collaboration. We also thank Yu Sawai, for the technical support with 16S ribosomal RNA gene sequencing method at Cykinso Co, and Techno Suruga Laboratory Co.,Ltd. For the support with SCFA analysis by gas chromatography. This research did not receive any specific grant from funding agencies in the public, commercial, or not-for-profit sectors. Dr. Naoko Hitosugi, MD, PhD, takes responsibility for the integrity of the data analysis.

\section{References}

1. Paugam C, CorvecS, Saint-Jean M, Le Moigne M, Khammari A, et al. (2017) Propionibacterium acnes phylotypes and acne severity: an observational prospective study. J Eur Acad Dermatol Venereol 31(9): e398-e399.

2. Yamazaki Y, Nakamura Y, Núñez G (2017) Role of the microbiota in skin immunity and atopic dermatitis. Allergol Int 66(4): 539-544.

3. Demirbaş A, Elmas ÖF (2020) The relationship between acne vulgaris and irritable bowel syndrome: A preliminary study. J Cosmet Dermatol 00: 1-5.

4. Deng Y, Wang H, Zhou J, Mou Y, Wang G, Xiong X (2018) Patients with Acne Vulgaris Have a Distinct Gut Microbiota in Comparison with Healthy Controls. Acta Derm Venereol 98(8): 783-790.

5. Maslowski KM, Mackay CR (2011) Diet, gut microbiota and immune responses. Nat Immunol 12(1): 5-9.

6. Putnam EE, Goodman AL (2020) B vitamin acquisition by gut commensal bacteria. PLoS Pathog 16(1): e1008208.

7. Hayashi N, Akamatsu H, Kawashima M, Acne Study Group (2008) Establishment of grading criteria for acne severity. J Dermatol 35(5): 255-260.

8. Duncan SH, Hold GL, Harmsen HJ, Stewart CS, Flint HJ (2002) Growth requirements and fermentation products of Fusobacterium prausnitzii, and a proposal to reclassify it as Faecalibacterium prausnitzii gen. nov., comb. nov. Int J Syst Evol Microbiol 52(6): 2141-2146.

9. Schwarz A, Bruhs A, Schwarz T (2017) The short-chain fatty acid sodium butyrate functions as a regulator of the skin immune system. J Invest Dermatol 137(4): 855-864.

10. Khan MT, Duncan SH, Stams AJ, van Dijl JM, Flint HJ, et al. (2012) The gut anaerobe Faecalibacterium prausnitzii uses an extracellular electron shuttle to grow at oxicanoxic interphases. ISMEJ 6(8): 1578-1585.

11. Kiefer D, Ali-Akbarian L (2004) A brief evidence-based review of two gastrointestinal illnesses: irritable bowel and leaky gut syndromes. Altern Ther Health Med 10(3): 22-30. 